\title{
Immunogenetics of some connective tissue disorders
}

\author{
Hasnaa Ahmed Abo El Wafa, ${ }^{1}$ Mohammad Abd Allah Mohammad, ${ }^{1}$ Shereen \\ Philip Aziz, ${ }^{1}$ Ahmed Sedky Mahmoud, ${ }^{1}$ Mustafa Adel Ahmed, ${ }^{1}$ AbeerAttia Ali ${ }^{2}$ \\ 1 Clinical \& Chemical Pathology Department, Faculty Of MedicineSohag University \\ 2 Resident of Blood Bank TemaHospital
}

\begin{abstract}
Connective tissue diseases (CTDs) are systemic autoimmune disorders characterized by a large spectrum of clinical features and multisystemic involvement. Connective tissue disease is any disease that has the connective tissues of the body as a target of pathology. Connective tissue is any type of biological tissue with an extensive extracellular matrix that supports, binds together, and protects organs. These tissues form a framework, or matrix, for the body, and are composed of two major structural protein molecules: collagen and elastin.

Autoimmunity involves the loss of normal immune homeostasis such that the organism produces an abnormal response to its own self tissue. The hallmark of autoimmune diseases generally involves the presence of self-reactive $\mathrm{T}$ cells, autoantibodies and inflammation
\end{abstract}

\section{Introduction}

Connective tissue diseases (CTDs) are systemic autoimmune disorders characterized by a large spectrum of clinical features and multisystemic involvement. CTDs include rheumatoid arthritis (RA), systemic lupus erythematosus (SLE), Sjögren syndrome (SS), systemic sclerosis (SSc), dermatomyositis (DM) and polymyositis (PM) However, undifferentiated connective tissue disease is observe in some patients who cannot be assigned to a single disease category. overlap syndrom is observe when manifestations from two or more CTDs are identified in the same patient. $^{(1)}$

\section{Systemic lupus erythromatosus}

Systemic lupus erythematosus (SLE) is a multisystem autoimmune disorder with complex etiology. Genetics plays an important role in lupus pathogenesis through its influence on clinical and autoantibody phenotype of the disease. ${ }^{(2)}$
The incidence rates of SLE range from approximately 1 to 10 per 100,000 personyears, and prevalence rates generally range from 20 to 70 per $100,000^{(3)}$

SLE, like many autoimmune diseases, affects females more frequently than males, at a rate of about 9 to $1^{(4)}$

The X chromosome carries immunological related genes, which can mutate and contribute to the onset of SLE. The Y chromosome has no identified mutations associated with autoimmune disease $^{(5)}$

Childhood-onset systemic lupus erythematosus generally presents between the ages of 3 and 15 and is four time more common in girls ${ }^{(6)}$

The disease appears to be more common in urban than rural areas. Sixty-five per cent of patients with SLE have disease onset between the ages of 16 and 55 years, $20 \%$ present before age 16 , and $15 \%$ after the age of $55^{(7)}$ 
The prevalence of late-onset systemic lupus erythematosus (SLE) diagnosed in patients over the age of 50 years is estimated at $10 \%$ to $20 \%$. ${ }^{(8)}$

\section{Aetiology}

The aetiology of SLE includes both genetic and environmental components with female sex strongly influencing pathogenesis. These factors lead to an irreversible break in immunological tolerance manifested by immune responses against endogenous nuclear antigens.

\section{Genetic factors}

Siblings of SLE patients are approximately 30 times more likely to develop SLE compared with individuals without an affected sibling.

For instance Tranducer And Activator Of Transcription 4Signal (STAT4) a genetic risk factor for rheumatoid arthritis and SLE, is associated with severe SLE. One of the key components of these pathways is TNFAIP3, which has been implicated in at least six autoimmune disorders, including SLE ${ }^{(9)}$

\section{Epigenetic effects}

The most well understood type of epigenetic factor is DNA methylation, which plays a role in a variety of human processes, such as $\mathrm{X}$ chromosome inactivation and certain cancers. Previous research has also implicated the importance of DNA methylation in SLE. Differences in the methylation status of genes may explain, at least in part, the discordance observed in some identical twins that are discordant for SLE. Epigenetic mechanisms may represent the missing link between genetic and environmental risk factors ${ }^{(9)}$

\section{Environmental factors}

Anumber of environmental triggering factors have been associated with SLE, including UV light and cigarette smoking, some of which trigger lupus through epigenetic mechanisms ${ }^{(10)}$

Epstein-Barr virus (EBV) and cytomegalovirus (CMV), have been linked to the pathogenesis of SLE by several reports). Commensal microbes residing inside the host, in return, have been shown to maintain and expand CD8+ memory $\mathrm{T}$ cells during CMV infection, supporting the notion that microbiota and CMV cooperatively augment immune activation $^{(11)}$

It is well established that certain drugs induce autoantibodies in a significant number of patients, most of whom do not develop signs of an autoantibody associated disease. These drugs may alter gene expression in CD4+ $\mathrm{T}$ cells by inhibiting DNA methylation and induce over-expression of LFA-1 antigen, thus promoting autoreactivity ${ }^{(\mathbf{1 2})}$

Antibiotics, which can remove gut bacteria, are known to trigger lupus flares. These include sulfa drugs such as trimethoprim- sulfamethoxazole (Septra), tetracycline-related antibiotics such as minocycline, and penicillin-related antibiotics such as amoxicillin ${ }^{(\mathbf{1 3})}$

Recently, several groups have found that metabolites produced by gut bacteria, especially butyrate produced by Clostridia, can promote the differentiation of regulatory $\mathrm{T}$ cells (Tregs) in the colon, spleen, and lymph nodes to suppress inflammation ${ }^{(14)}$

\section{Hormonal factors}

More than $90 \%$ of systemic lupus erythematosus (SLE) patients are female, suggesting an important role for sex hormones. Epidemiological data suggests both pregnant and postmenopausal women are more susceptible to developing SLE ${ }^{(5)}$

Disease activity in female SLE is associated with low testosterone, androstenedione, anddehydroepiandrostero ne (DHEA). Moreover, immune cells express estrogen receptors, and lymphocyte activation by estrogen is associated with SLE disease activity ${ }^{(15)}$

\section{Rheumatoid arthritis}

Rheumatoid arthritis (RA) is a chronic, systemic autoimmune inflammatory disease that affects approximately $1 \%$ of the population. Initial symptoms include joint swelling, stiffness, and tenderness, which are all causes of disability. The diagnosis of RA is based on patient history of joint pain and stiffnes ${ }^{(16)}$ 
RA affects between 0.5 and $1 \%$ of adults in the developed world with between 5 and 50 per 100,000 people newly developing the condition each year ${ }^{(17)}$

The incidence rate ratio among ever smoking patients was 1.96 after 30 packyears, and 1.034 per year of smoking implying a doubling of risk after 20 years regardless of sex and smoking intensity ${ }^{(\mathbf{1 8})}$

\section{Genetic and Environmental Factors}

Rheumatoid arthritis involves a complex interplay among genotype, environmental triggers, and chance. Twin studies implicate genetic factors in rheumatoid arthritis, with concordance rates of 15 to $30 \%$ among monozygotic twins and 5\% among dizygotic twins ${ }^{(19)}$

The long-established association with the human leukocyte antigen (HLA)-DRB1 locus has been confirmed in patients who are positive for rheumatoid factor or ACPA. ${ }^{(20)}$

These findings suggest that some predisposing T-cell repertoire selection, antigen presentation, or alteration in peptide affinity has a role in promoting autoreactive adaptive immune responses. (21)

Genetic risk factors for ACPA-negative disease appear to be not less important than those for ACPA-positive disease. However, they are less well established and involve different HLA alleles (e.g., HLA DRB1*03), interferon regulatory factors (e.g., interferon factor 5), and lectin-binding proteins (e.g., C-type lectin domain family 4 member A) ${ }^{\text {(22) }}$

Moreover, smoking and HLA-DRB1 alleles synergistically increase one's risk of having ACPA. ${ }^{(23)}$

Several citrullinated self-proteins are recognized in anti-CCP assays, including $\alpha$-enolase, keratin, fibrinogen, fibronectin, collagen, and vimentin ${ }^{(24)}$

Infectious agents (e.g., Epstein-Barr virus, cytomegalovirus, proteus species, and Escherichia coli) and their products (e.g., heat-shock proteins) have long been linked with rheumatoid arthritis. ${ }^{(25)}$

Finally, the gastrointestinal microbiome is now recognized to influence the development of autoimmunity in articular models, and specific clinical bacterial signatures that are associated with autoantibody positive rheumatoid arthritis are emerging (26)

\section{Systemic sclerosis}

Systemic sclerosis (SSc) (scleroderma) is a complex autoimmune disease that clinically manifests as progressive fibrosis of the skin and internal organs. Anticentromere antibodies (ACAs), antitopoisomerase antibodies (ATAs), and anti-RNA polymerase III antibodies (ARAs) are three mutually exclusive SScassociated autoantibodies that correlate with distinct clinical subsets characterized by extent of cutaneous involvement and pattern of organ involvement ${ }^{(27)}$

Scleroderma is a chronic sclerosing disease of the connective tissues. Hidebound skin is an important and characteristic feature of this disease ${ }^{(28)}$

Scleroderma exists in two forms: morphea (circumscribed scleroderma) and generalized/progressive(diffuse

scleroderma). ${ }^{(29)}$

Systemic sclerosis is a rare disease. Systemic scleroderma has an incidence of $0.3-2.8 / 100,000 /$ year and a prevalence of $1-15 / 100,000$. The distinct female predominance is seen in the female: male ratio of $3: 1^{(30)}$

\section{Type of Systemic Sclerosis Diffuse Scleroderma}

Affects the skin as well as the heart, lungs, GI tract, and kidneys.

\section{Limited Scleroderma}

Mostly affects the skin of the face, neck and distal elbows and knees and late in the disease causes isolated pulmonary hypertension. CREST syndrome (Calcinosis, Raynaud's phenomenon, Esophageal dysfunction, Sclerodactyly, Telangiectasias) is associated with limited scleroderma ${ }^{(31)}$

The diffuse form of systemic sclerosis (DSSc) occurs equally in males and females. The limited form of systemic sclerosis (LSSc) has a strong female predominance, with a female-to-male ratio of 10:1. ${ }^{(32)}$ 
Systemic sclerosis usually appears in women aged 30-40 years, and it occurs in slightly oldermen.Pulmonary hypertension leads to $12 \%$ of systemic sclerosis-related deaths.

Lung fibrosis and heart changes are responsible for $9 \%$ of systemic sclerosisrelated deaths. ${ }^{(33)}$

\section{Family and twin studies}

The disease can occur in families in which a member has already been diagnosed with SSc or another autoimmune disease. Having a sibling with SSc is one of the highest risk factors (34)

\section{Candidate-gene studies}

Multiple candidate-gene studies of SSc have been carried out. Most often, the candidate genes and single-nucleotide polymorphisms (SNPs) were selected on the basis of their involvement in the susceptibility to other autoimmune diseases ${ }^{(35)}$

\section{Genome-wide association studies}

GWAS identifiedthat IRF8 is specifically associated with lcSSc. The same study also demonstrated that HLA-DQB1 is associated with anti-centromere autoantibodies (ACA) positivity, whereas HLA-DPA1/B1 was associated with antitopoisomerase autoantibodies (ATA) positivity. Less discriminating was NOTCH4, which was associated with the presence of both ACA and ATA ${ }^{(36)}$

GWAS show that $\mathrm{T}$ cell signalling and interferon signalling pathways are involved in SSc susceptibility, and reveal roles in apoptosis, DNA or RNA degradation and autophagy ${ }^{(37)}$

\section{The role of epigenetics in SSc}

The environment can affect the breakage of immune tolerance and the development of fibrosis and $\mathrm{SSc}$ in certain genetic backgrounds. This concept is supported by numerous studies indicating that different environmental factors, including occupational exposure to silica dust, vinyl chloride or drugs such as bleomycin, can induce SSc-like symptoms ${ }^{(38)}$

Interestingly, no clear association between smoking and the risk of developing SSc has been

established.Potential mechanisms for environmentally induced systemic autoimmunity include interference with immune tolerance, activation of the immune system, induction of genetic alterations and dysregulation of epigenetic patterns ${ }^{(39)}$

\section{Dermatomyositis and polymyositis}

Dermatomyositis (DM) and polymyositis (PM) are autoimmune myopathies characterized clinically by proximal muscle weakness, muscle inflammation,extramuscularmanifestations and frequently, the presence of autoantibodies. Although there is some overlap, DM and PM are separate diseases with different pathophysiological mechanisms ${ }^{(40)}$

The estimated incidence of dermatomyositis is 9.63 cases per million population. The estimated incidence of amyopathicdermatomyositis (AMD) is 2.08 cases per million ${ }^{(41)}$

\section{Immune mechanisms in PM and DM}

The strongest support for an immunemediated myopathy is the presence of cellular infiltrates of both the adaptive and innate immune systems in muscle biopsies and the frequent presence of autoantibodies. $^{(\mathbf{4 2})}$

\section{Autoantibodies}

To date a majority of patients with PM and DM has at least one myositis specific antibody (MSA) if sensitive techniques to identify autoantibodies are utilized. Other autoantibodies can also be found, so-called myositis-associated autoantibodies (MAAs), which may also be present in other autoimmune diseases such as SLE and SS. The most frequently present MAAs in PM and DM are anti-SSA or anti Ro-52 and anti-PMScl ${ }^{(\mathbf{4 3})}$

\section{Sjögren's syndrome}

Sjögren's syndrome (SS), a systemic autoimmune disease, is characterized by inflammation of exocrine tissues accompanied by a significant loss of their secretory function. Clinical symptoms develop late and there are no diagnostic tests enabling early diagnosis of SS ${ }^{(44)}$ 
Sjögren syndrome affects $0.1-4 \%$ of the population. This wide range, in part, reflects the lack of uniform diagnostic criteria $^{(\mathbf{4 5})}$

The female-to-male ratio of Sjögren syndrome is 9:1. Sjögren syndrome can affect individuals of any age but is most common in elderly people. Onset typically occurs in the fourth to fifth decade of life

One of the consequences of the stimulation of innate immunity is the activation of nuclear factor $\kappa \mathrm{B}(\mathrm{NF} \kappa \mathrm{B})$, which can occur in a number of different cell types. In salivary gland epithelial cells from patients with $\mathrm{pSS}$, hyperactivation of $\mathrm{NF} \kappa \mathrm{B}$ has been associated with decreased expression of one of the regulators of NFKB activation, A20 (also known as TNF $\alpha$-induced protein 3 [TNFAIP3]) ${ }^{(46)}$

Furthermore, the first GWAS in pSS identified an association between this disease and a gene (TNIP1) encoding TNFAIP3-interacting protein 1, a protein that interacts with A20 and is involved in the regulation of $\mathrm{NF} \kappa \mathrm{B}$ activation. ${ }^{(47)}$

\section{References}

1- Iaccarino L, Ghirardello A, CanovaM, ZenM, Bettio S and Nalotto L. (2011): Anti-annexins autoantibodies: their role as biomarkers of autoimmune diseases. 10:553-8.

2- PanneerDevaraju, ReenaGulati, Paul T, Antony CB and Mithun. (2015): Susceptibility to SLE in South Indian Tamils may be influenced by genetic selection pressure on TLR2 and TLR9 genes. Volume 64, Issue 1, March 2015, Pages 123-126

3- Somers EC, Marder $\mathrm{W}$ and Cagnoli $\mathrm{P}$. (2014): Population-based incidence and prevalence of systemic lupus erythematosus: the Michigan Lupus Epidemiology and Surveillance program. Arthritis Rheumatol (Hoboken NJ) 66(2):369-378

5- Tsokos GC. (2011): "Systemic lupus erythematosus". N. Engl. J. Med. 365 (22): 2110-21

4- Murphy, G; Isenberg, D (2013): "Effect of gender on clinical presentation in systemic lupus erythematosus.". Rheumatology (Oxford, England) 52 (12): 2108-15

6- Borgia RE and Silverman ED (2015): "Childhood-onset systemic lupus erythematosus: an update.". Current opinion in rheumatology 27 (5): 483-9

7- Bertsias GK, Salmon JE and Boumpas DT. (2010): Therapeutic opportunities in systemic lupus erythematosus: state of the art and prospects for the new decade. Ann Rheum Dis. 69:1603-1611

8- Jeleniewicz R, Suszek D and Majdan M. (2015): Clinical picture of late-onset systemic lupus erythematosus in a group of Polish patients, Pol Arch Med Wewn. 125(7-8):538-44. Epub 2015 Jun 15

9-George Bertsias, RicardCervera and Dimitrios T Boumpas, (2012): EULAR Textbook on Rheumatic Diseases, Systemic Lupus Erythematosus: Pathogenesis and Clinical Feature. ch 20,479-505

10- Javierre BM, Fernandez AF and Richter J. (2010): Changes in the pattern of DNA methylation associate with twin discordance in systemic lupus erythematosus. Genome Res. 20:170-179

11- Somers EC and Richardson BC. (2014): Environmental exposures, epigenetic changes and the risk of lupus. Lupus. 23(6):568-76.

12- Rasmussen NS, Draborg AH, Nielsen CT, Jacobsen S and Houen G. (2015): Antibodies to early EBV, CMV, and HHV6 antigens in systemic lupus erythematosus patients. Scand J Rheumatol. 44(2):143-9

13- Mak A, Tay S (2014): Environmental factors, toxicants and systemic lupus erythematosus. Int J MolSci 15(9):16043-16056.

14- Singh N, Gurav A, Sivaprakasam S, Brady E, Padia R and Shi H. (2014): Activation of Gpr109a, receptor for niacin and the commensal metabolite butyrate, suppresses colonic inflammation and carcinogenesis. Immunity. 40(1):128-39.

15- Kassi E and Moutsatsou P. (2010): Estrogen receptor signaling and its relationship to cytokines in systemic 
lupus erythematosus. J Biomed Biotechnol. 2010: 317452..

16- Agarwal SK. (2011): Core management principles in rheumatoid arthritis to help guide managed care professionals. NovDec;17(9 Suppl B): S03-8.

17- Scott DL, Wolfe F and Huizinga TW. (2010): "Rheumatoid arthritis". Lancet 376 (9746): 1094-108.

18- Svendsen AJ, Junker P, Houen G, Kyvik KO, Nielsen C, Skytthe A, Holst R. (2016): Incidence of chronic persistent rheumatoid arthritis and the impact of smoking. Arthritis Care Res (Hoboken). Jul 7

19- MacGregor AJ, Snieder H and Rigby AS. (2010): Characterizing the quantitative genetic contribution to rheumatoid arthritis using data from twins. Arthritis Rheum. 43:30-7.

20- Gregersen PK, Silver $J$ and Winchester RJ. (2011): The shared epitope hypothesis: an approach to understanding the molecular genetics of susceptibility to rheumatoid arthritis. Arthritis Rheum. 30:1205- 13

21- De Almeida DE, Ling S, Pi X, HartmannScruggs AM, Pumpens $\mathrm{P}$ and Holoshitz J. (2010): Immune dysregulation by the rheumatoid arthritis shared epitope. J Immunol. 185:1927-34..

22- Klareskog L, Ronnelid J, Lundberg K, Padyukov L and Alfredsson L. (2008): Immunity to citrullinated proteins in rheumatoid arthritis. Annu Rev Immunol. 26:651-75.

23- De Rycke L, Peene I and Hoffman IE. (2011): Rheumatoid factor and anticitrullinated protein antibodies in rheumatoid arthritis: diagnostic value, associations with radiological progression rate, and extraarticular manifestations. Ann Rheum Dis. 63:1587-93.

24- Mahdi $H$, Fisher $B A$ and Kallberg $H$. (2009): Specific interaction between genotype, smoking and autoimmunity to citrullinated -alpha-enolase in the etiology of rheumatoid arthritis. Nat Genet. 41:1319- 24

25- Wegner N, Wait R and Sroka A. (2010): Peptidylargininedeiminase
Porphyromonasgingivaliscitrullinates

human fibrinogen and $\alpha$-enolase: implications for autoimmunity in rheumatoid arthritis. Arthritis Rheum. 62:2662-72..

26- Scher JU, Ubeda $\mathrm{C}$, Pillinger $\mathrm{MH}$, et al. (2010): Characteristic oral and intestinal microbiota in rheumatoid arthritis (RA): a trigger for autoimmunity? Arthritis Rheum. 62:Suppl:1390.

27-Wu M, Schneider DJ, Mayes MD, Assassi S, Arnett FC, Tan FK, Blackburn MR and Agarwal SK. (2012): The role of type 1 interferon in systemic sclerosis. Jun;132(6):1605-14.

28- Vikram B.(2013): A rare case of hidebound disease with dental implications. Dent Res J (Isfahan). 10(4):556-61.

29- Careta MF and Romiti R. (2015): Localized scleroderma: clinical spectrum and therapeutic update. An Bras Dermatol. 90(1):62-73.

30- Michael. (2012): Sticherling Systemic sclerosis - dermatological aspects. Part 1: Pathogenesis, epidemiology, clinical findings. Volume 10, Issue 10 October. Pages 705-716

31- Tyndall A and Fistarol S. (2013): The differential diagnosis of systemic sclerosis. CurrOpinRheumatol. 25:692-9.

32- Nguyen C, Berezne A, Baubet $T$, et al. (2011): Association of Gender with Clinical Expression, Quality of Life, Disability, and Depression and Anxiety in Patients with Systemic Sclerosis. PLoS One. Mar 9. 6(3): e17551.

33- Ungprasert $\mathrm{P}$, Srivali $\mathrm{N}$ and Kittanamongkolchai W. (2015): Systemic sclerosis and risk of venous thromboembolism: A systematic review and meta-analysis. Mod Rheumatol. Apr 7. 1-17

34- Arora-Singh RK. (2010): Autoimmune diseases and autoantibodies in the first degree relatives of patients with systemic sclerosis. J. Autoimmun. 35, 52-57.

35- Broen JC, Coenen M J, and Radstake T R. (2012): Genetics of systemic sclerosis: an update. Curr. Rheumatol. Rep. 14, 1121. 
36- Gorlova O. (2011): Identification of novel genetic markers associated with clinical phenotypes of systemic sclerosis through a genome-wide association strategy. PLoS Genet. 7, e1002178.

37- Mayes MD. (2014): Immunochip analysis identifies multiple susceptibility loci for systemic sclerosis. Am. J. Hum. Genet. 94, 4761

38- Ballestar E. (2011): Epigenetic alterations in autoimmune rheumatic diseases. Nat. Rev. Rheumatol. 7, 263-271.

39- Marie I. (2014): Prospective study to evaluate the association between systemic sclerosis and occupational exposure and review of the literature. Autoimmun. Rev. 13, 151-156.

40-Mammen

AL.

(2010):

Dermatomyositis and polymyositis: Clinical presentation, autoantibodies, and pathogenesis. Jan;1184:134-53.

41- Bendewald MJ, Wetter DA, Li $X$ and Davis MD. (2010): Incidence of dermatomyositis and clinically amyopathicdermatomyositis: apopulation-based study in Olmsted County, Minnesota. Arch Dermatol. Jan. 146(1):26-30.
42- Paulius Venalis and Ingrid E. (2014): Lundberg. Immune mechanisms in polymyositis and dermatomyositis and potential targets for therapy. Rheumatology Journal. 53:397_405.

43- Betteridge ZE, Gunawardena $\mathrm{H}$ and McHugh NJ. (2011): Novel autoantibodies and clinical phenotypes in adult and juvenile myositis. Arthritis Res Ther. 13:209.

44- Delaleu N, Nguyen CQ, Peck AB and Jonsson R. (2011): Sjögren's syndrome: studying the disease in mice. Jun 13;13(3):217.

45- Helmick CG, Felson DT and Lawrence RC. (2008): Estimates of the prevalence of arthritis and other rheumatic conditions in the United States. Part I. Arthritis Rheum. Jan. 58(1):15-25.

46- Sisto M. (2011): A failure of TNFAIP3 negative regulation maintains sustained NF- $\kappa \mathrm{B}$ activation in Sjögren's syndrome. Histochem. Cell Biol. 135, 615-625.

47- Lessard CJ. (2013): Identification of multiple Sjögren's syndrome susceptibility loci [abstract OP0020]. Ann. Rheum. Dis. 72 (Suppl. 3), 54. 
